\title{
Identifying Key Issues and Potential Solutions for Integrated Arrival, Departure, Surface Operations by Surveying Stakeholder Preferences
}

\author{
Bimal Aponso ${ }^{1}$, Richard Coppenbarger ${ }^{2}$, Yoon Jung ${ }^{3}$, and Leighton Quon ${ }^{4}$ \\ NASA Ames Research Center, Moffett Field, CA 94035 \\ Gary Lohr ${ }^{5}$, and Neil O'Connor ${ }^{6}$ \\ NASA Langley Research Center, Hampton, VA 23681 \\ and \\ Shawn Engelland ${ }^{7}$ \\ NASA Ames Research Center, Fort Worth, TX, 76155
}

\begin{abstract}
NASA's Aeronautics Research Mission Directorate (ARMD) collaborates with the FAA and industry to provide concepts and technologies that enhance the transition to the nextgeneration air-traffic management system (NextGen). To facilitate this collaboration, ARMD has a series of Airspace Technology Demonstration (ATD) sub-projects that develop, demonstrate, and transitions NASA technologies and concepts for implementation in the National Airspace System (NAS). The second of these sub-projects, ATD-2, is focused on the potential benefits to NAS stakeholders of integrated arrival, departure, surface (IADS) operations. To determine the project objectives and assess the benefits of a potential solution, NASA surveyed NAS stakeholders to understand the existing issues in arrival, departure, and surface operations, and the perceived benefits of better integrating these operations. NASA surveyed a broad cross-section of stakeholders representing the airlines, airports, air-navigation service providers, and industry providers of NAS tools. The survey indicated that improving the predictability of flight times (schedules) could improve efficiency in arrival, departure, and surface operations. Stakeholders also mentioned the need for better strategic and tactical information on traffic constraints as well as better information sharing and a coupled collaborative planning process that allows stakeholders to coordinate IADS operations. To assess the impact of a potential solution, NASA sketched an initial departure scheduling concept and assessed its viability by surveying a select group of stakeholders for a second time. The objective of the departure scheduler was to enable flights to move continuously from gate to cruise with minimal interruption in a busy metroplex airspace environment using strategic and tactical scheduling enhanced by collaborative planning between airlines and service providers. The stakeholders agreed that this departure concept could improve schedule predictability and suggested several key attributes that were necessary to make the concept successful. The goals and objectives of the planned ATD-2 sub-project will incorporate the results of this stakeholder feedback.
\end{abstract}

${ }^{1}$ Aerospace High-Density Operations Branch, AIAA Associate Fellow

${ }^{2}$ Flight Trajectory Dynamics and Controls Branch, AIAA Associate Fellow

${ }^{3}$ Aerospace High-Density Operations Branch, AIAA Senior Member

${ }^{4}$ Airspace Technology Demonstrations Project Manager, AIAA Associate Fellow

${ }^{5}$ Crew Systems \& Aviation Operations Branch

${ }^{6}$ Crew Systems \& Aviation Operations Branch

${ }^{7}$ Systems Modeling and Optimization Branch, AIAA Senior Member 


\section{Introduction}

The "Next Generation Air Transportation System," known as NextGen, is a comprehensive transformation of the National Airspace System (NAS) to a system that will be safer, more reliable, more efficient, and which will reduce the impact of aviation on the environment. The transition to NextGen is vital to improving system performance, meeting continued growth in air traffic, and increasing the Nation's mobility to support economic progress. The Federal Aviation Administration (FAA) estimates that by 2020, NextGen improvements will reduce delays by 41 percent, reduce cumulative carbon dioxide emissions by 16 million metric tons, and reduce fuel use by 1.6 billion gallons in cumulative. Taken together, these and other efficiency improvements will provide an estimated $\$ 38$ billion in cumulative benefits to aircraft operators, the traveling public, and the FAA by 2020 (Ref. 1).

The Aeronautics Research Mission Directorate (ARMD) of the National Aeronautics and Space Administration (NASA) is collaborating with the FAA and other industry partners to enhance the transition to NextGen by developing advanced automation concepts and tools that provide air traffic controllers, pilots, and other airspace users with more accurate real-time information and advisories about the nation's traffic flow, weather, and routing. Improving the precision of these information sources and intelligently combining them to enable better decision-making in the NAS are key enablers of NextGen. NASA's NextGen related programs research and develop new concepts and technologies, evaluates them in an operationally relevant environment, demonstrates their feasibility and benefits, and transitions them to an external organization (primarily the FAA) for implementation in the NAS. Examples of NASA technologies that have completed this cycle include Traffic Management Advisor (TMA) (Ref. 2) and the Efficient Descent Advisor (EDA)/3D Path Arrival Management (3D-PAM) concept (Ref. $3)$.

To streamline this process, ARMD created the Airspace Technology Demonstration (ATD) Project that builds upon fundamental research conducted by NASA and its partners and demonstrates integrated, domain focused solutions for NAS operations. The goal of this project is to accelerate the process of maturing new concepts and technologies and transition them to stakeholders while demonstrating their feasibility and quantifying their benefit to the NAS. The current and planned ATD sub-projects are:

- ATD-1: Interval Management - Terminal Area Precision Scheduling and Spacing.

- ATD-2: Integrated Arrival/Departure/Surface Operations (IADS) Metroplex Traffic Management.

- ATD-3: Applied Traffic Flow Management.

- TASA: Technologies for Airplane State Awareness

The TASA sub-project seeks to mitigate flight crew loss of airplane state awareness as a causal factor in commercial aviation accidents and incidents by developing cockpit systems with predictive algorithms, models for aircraft stall performance, and flight crew training methods that may be transitioned to the aviation community together with the Commercial Aviation Safety Team (CAST).

The ATD-1 sub-project enhances the initial TMA concept and improves the precision and throughput of terminal arrivals by developing and operationally demonstrating an integrated set of NASA arrival management software technologies for planning and executing efficient arrival operations in the terminal environment of a highdensity airport. The key enabler is NASA's Terminal Sequencing and Spacing (TSS) technology (Ref. 4) that was transferred to the FAA and will be implemented in the NAS in the near future. The ATD-2 and ATD-3 sub-projects are in their advanced and preliminary planning stages, respectively. ATD-2 will investigate improving the efficiency of arrival, departure, and surface operations while ATD-3 will investigate efficient routing concepts for en-route weather avoidance and oceanic operations. This paper describes the initial planning process for ATD-2.

NASA's research portfolio includes several promising technologies for improving surface and departure operations that are in relatively advanced stages of development with active stakeholder partnership. As a result, NASA decided to explore the potential for improving IADS operations using these technologies in the ATD-2 subproject. The first step in this process, however, was to clearly understand the IADS technology needs from the stakeholder's point-of-view. As part of planning the project and setting its goals, therefore, NASA needed to identify areas in the current arrival, departure, surface operational environment where an integrated solution could offer the greatest benefit to the stakeholders. With this understanding, the ATD-2 sub-project could be structured to develop and demonstrate an integrated concept that would be valued and accepted by these stakeholders. To understand the critical issues related to IADS operations, NASA reached out to relevant stakeholders and gathered information and insights that enhanced NASA's understanding of the challenges faced by these stakeholders and identified the benefit (value) of potential solutions. The results of the stakeholder survey were combined with a literature survey 
on IADS issues and previous research, sorted, categorized, and converted to project goals and technical objectives through the process illustrated in Fig. 1.

This paper presents the stakeholder survey process, results, and an initial discussion of project goals that were distilled from this information. NASA is presently formally planning and formulating the ATD-2 sub-project to address the goals derived from the information provided by the stakeholders.

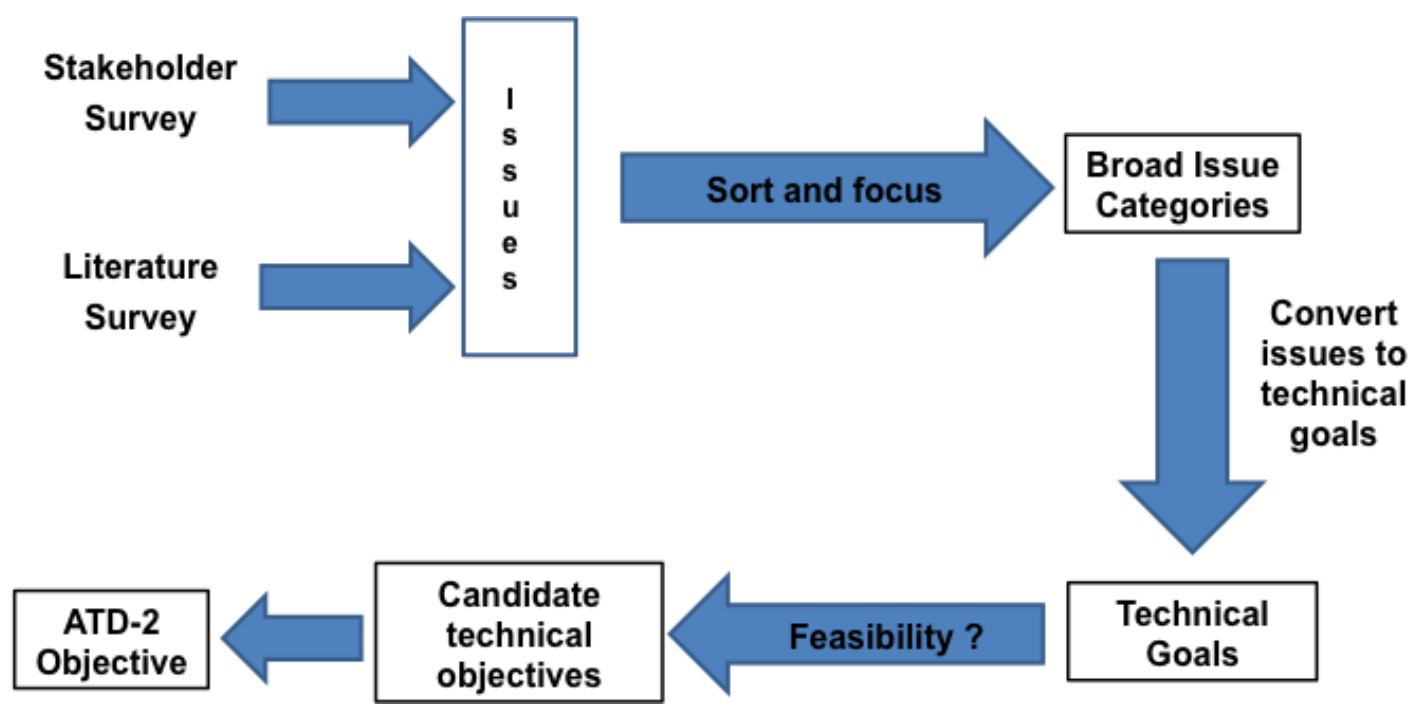

Figure 1. Process for developing project objectives from stakeholder feedback.

\section{Background}

Arrival, departure, surface operations involve a complex interplay between stakeholders. For an integrated solution to be successful in this environment, it must address the economic and operational issues that influence this interplay. Before approaching the stakeholders to understand their viewpoint on these issues, a preliminary literature survey was conducted to get a broad understanding of this environment. This survey identified two basic factors that need to be considered by an IADS solution. These are: the economic factors that influence airline arrival, departure, surface operations, and the technical landscape that a potential IADS solution needs to integrate with. These are introduced and discussed briefly here to set a context for the stakeholder survey that is discussed later.

\section{A. Relevant Economic Factors - Airline Block Times and On-Time Metrics}

Efficiencies in NAS operations, and IADS operations in particular, will be measured primarily in economic terms. It was, therefore, important to understand the basic economic factors driving one of the major stakeholder groups - the commercial airlines. A key measure of airline cost performance is block time (Ref. 5). The block time is the elapsed time for a flight when the airline's fixed asset (the airplane) is earning revenue by transporting passengers. To earn this revenue, an airline incurs direct operating costs including primarily crew and fuel costs that scale with block time. Typically, block time is determined as the time between the cabin door closing at departure and opening on arrival (Ref. 5). On any flight, there are four distinct event-time signals that are transmitted from the aircraft to the airline (Ref. 6). These are depicted in Fig. 2 and defined as: OUT (cabin door close/parking brake release), OFF (weight off landing gear), ON (weight on landing gear), and IN (cabin door open/parking brake set). Block time is defined as the elapsed time between the OUT and IN signals (Ref. 5). A key airline cost factor that is linked to scheduled or actual block time is the flight crew compensation rate (Ref. 5). 


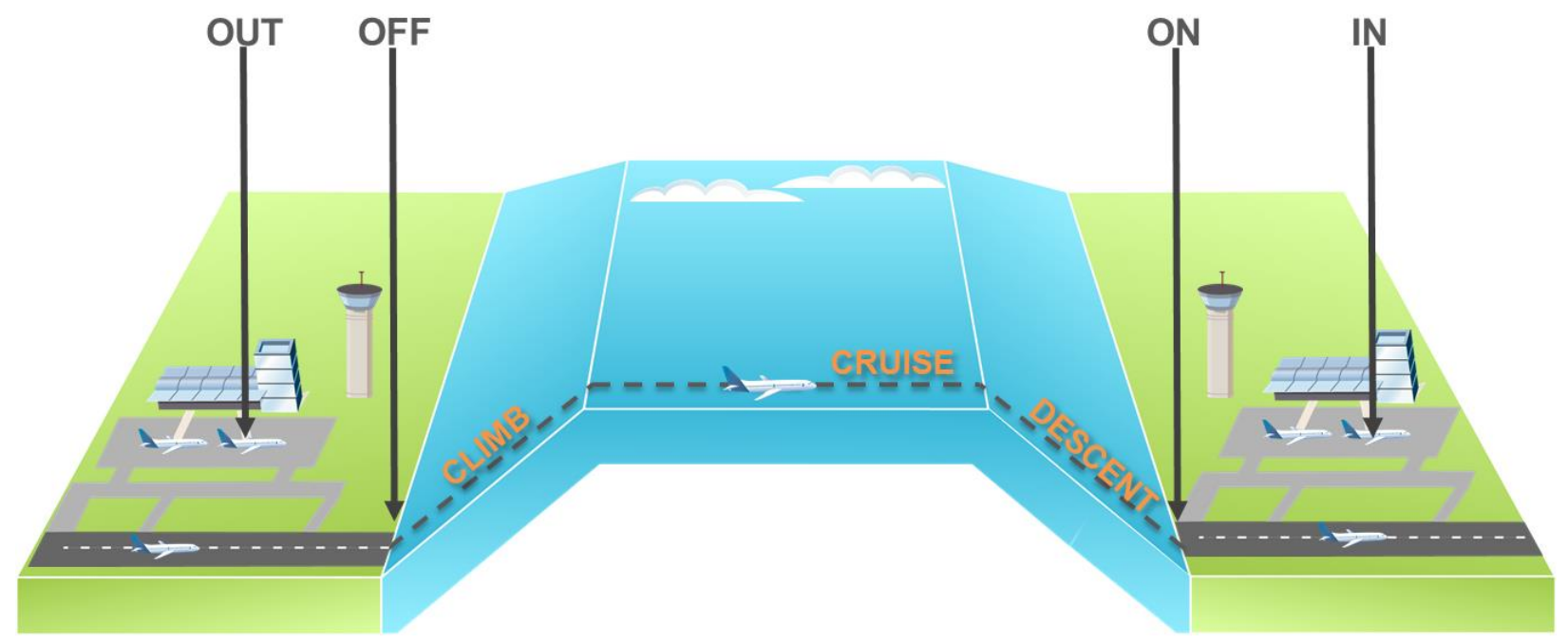

Figure 2. Event-time communications from a commercial flight.

Airlines maintain comprehensive databases of actual historical block times for each city-pair within their route network. These databases are extremely sophisticated and include all the possible variables that influence block time, including type of aircraft, day-of-year, time-of-day, seasons, typical air-traffic constraints, airport issues, etc (Ref. 5). This historic data is used to forecast future block times (known as scheduled block times) for planning route networks, flight schedules, revenues/costs, and business strategy.

A report by an industry/air-navigation service provider focus group explains that air-traffic management (ATM) performance is critical to how airlines plan and operate their schedules and proposes several metrics that assesses the effect of the ATM system on airline economic performance (Ref. 7). Flight schedule delay and predictability are two of the key metrics that are defined. The discussion surrounding these metrics in Ref. 7 indicates the mechanism by which increased delay and reduced predictability affect costs and increases scheduled block times. Ref. 7 also points out that scheduled block time for flights among the 29 hub airports in the U.S. increased by an average of 1.25 minutes from 1993 to 1997, and discusses the adverse effect of poor predictability on the integrity of an airline's network, schedule, and productivity.

The MIT International Center for Air Transportation (ICAT) delved into actual block times and investigated the causes of delays on US domestic routes by assessing delays incurred at each "segment" of block time - taxi out, departure, en-route, arrival, and taxi-in (Ref. 8). This analysis showed how airlines tended to be conservative in determining their scheduled block times - lengthening the scheduled flight time (scheduled block time) by adding "buffer" times to increase the likelihood that the aircraft will arrive at, or before, the scheduled arrival time and, thereby, improve the reliability of their schedules (Ref. 8).

Scheduled block times are also indirectly influenced by the "on-time" data that are voluntarily reported by the airlines to the US Department of Transportation's (USDOT) Bureau of Transportation Statistics (BTS). These data assesses airline performance against their schedules - a flight is counted as "on time" if its actual arrival or departure time (from the gate) is less than 15 minutes after the published scheduled time. Flights arriving early are counted as on time. Airline on-time performance statistics based on these data are compiled and published monthly by the BTS and are used by non-governmental organizations to rank airline schedule performance. As a result, performing well on these metrics is a priority for airlines.

\section{B. Relevant Technical Factors - Existing Concepts and Technologies}

The FAA has several programs that are designed to introduce new technologies and improve the efficiency of the air-traffic management system. These technology programs typically serve areas of the NAS where an aircraft is managed by air-traffic control (ATC) - from the time the aircraft enters an active taxiway to the time it leaves it (known as the "movement area"). Airport gate and ramp areas are not usually controlled by ATC and are known as "non-movement areas." They are usually managed by the airline or airport authority. 
Many of the upgrades to the ATM system are consolidated under three large FAA Decision Support System programs that introduce new technologies in discrete "builds" as technologies and funding becomes available (Ref. 1). These three programs are:

- $\quad$ Time Based Flow Management (TBFM) - is an existing system that facilitates time-based metering for managing arrivals that is based on NASA's TMA technology (Ref. 2). The TSS technology developed under NASA's ATD-1 project (Ref. 4) will be introduced into future builds of this system. Future builds are also expected to include NASA's EDA (Ref. 3) and Precision Departure Release Capability (PDRC - Ref. 9) technologies.

- Terminal Flight Data Manager (TFDM) - is a proposed system that will improve airport surface and terminal area operations by integrating airport surveillance, flight data, and automation systems available to ATC in the surface area. A Concept of Operations (ConOps) for TFDM is available (Ref. 10) and procurement of an initial capability is being planned. Future builds of TFDM are expected to include time-based management of departures.

- Traffic Flow Management System (TFMS) - this system is the FAA's primary tool for traffic flow management. It gathers and processes flight plan and schedule information as well as NAS capacity constraints to enable the demand on the NAS to be assessed and compared with the available constraints. Future builds are expected to include automation and decision-support tools to alleviate NAS constraints.

In addition, the FAA's System Wide Information Management (SWIM) initiative supports these and other NextGen programs by improving architecture and infrastructure to enable interoperability and data sharing between NAS stakeholders (Ref. 11).

In parallel with these programs, the FAA is studying concepts and technologies to include in future builds of these systems. One such concept promotes collaborative planning for airport departure operations using mechanisms and procedures similar to those used in Collaborative Decision Making (CDM) for strategic traffic flow management. The basic precepts of collaborative planning are that better information will lead to better decisionmaking, and that tools and procedures need to be in place to enable air navigation service providers and the flight operators to more easily respond to changing conditions. The Surface CDM ConOps (Ref. 10), developed by the FAA and industry stakeholders, is founded on the premise that access to real-time aircraft position data on the airport surface coupled with timely sharing of accurate operational data among stakeholders affords the opportunity to better predict and manage demand on the airport surface. When implemented, the Surface CDM ConOps is expected to change the ATC's current "first-come, first-served" operating paradigm for managing surface traffic by incorporating strategic planning through information sharing and collaborative planning between airlines and service providers. The TFDM program encompasses the concepts in the Surface CDM ConOps (Ref. 10).

The FAA also sponsored a broader investigation on improving IADS operations. This investigation, summarized in Ref. 12, found that developing and implementing an integrated schedule for arrivals and departures at an airport could improve IADS operations. This integrated schedule would be updated using data sharing between the key stakeholders. The concept called for incorporating airspace and surface constraints in the integrated schedule. An integrated scheduler would allow airlines to absorb delay in the non-movement area (preferably at the gate) as opposed to the movement area where parked aircraft might contribute to congestion.

Airlines and airports also use industry provided customized solutions to assess arrival/departure demand at airports and use this information to manage flights at the gate and maximize schedule integrity at specific airports. At a few airports in the US, these customized solutions use data sharing and collaborative planning to improve overall throughput while allowing airlines to maintain schedule integrity (Ref. 13). In the US, these systems manage flights in the non-movement area and their information is not used by the ATC system, which operates on the "firstcome, first-served" operating paradigm.

\section{Stakeholder Survey Process}

A preliminary examination of IADS operations and technologies identified four broad stakeholder categories. These are:

- Airlines;

- Airports;

- Air Navigation Service Providers (ANSPs); and

- Industry service/product providers. 
The primary goal of the survey was to uncover all the key issues faced by stakeholders in IADS operations - thus, emphasis was placed on the breadth of understanding of the issues as opposed to depth on specific operational issues. Also, an understanding of the economic and operational impact of any issue to a stakeholder was critical, therefore, particular emphasis was placed on identifying sources with knowledge of operational and business/financial aspects of their companies or organizations. Figure 3 shows the companies and organizations that provided information for the stakeholder survey. They included major US commercial passenger and cargo carriers; airport authorities of major US airports; groups within the FAA with responsibility for surface and terminal operations, operational infrastructure planning, and NextGen implementation; and industry providers of aircraft, avionics, and software/hardware tools that support airport surface and terminal area traffic management.

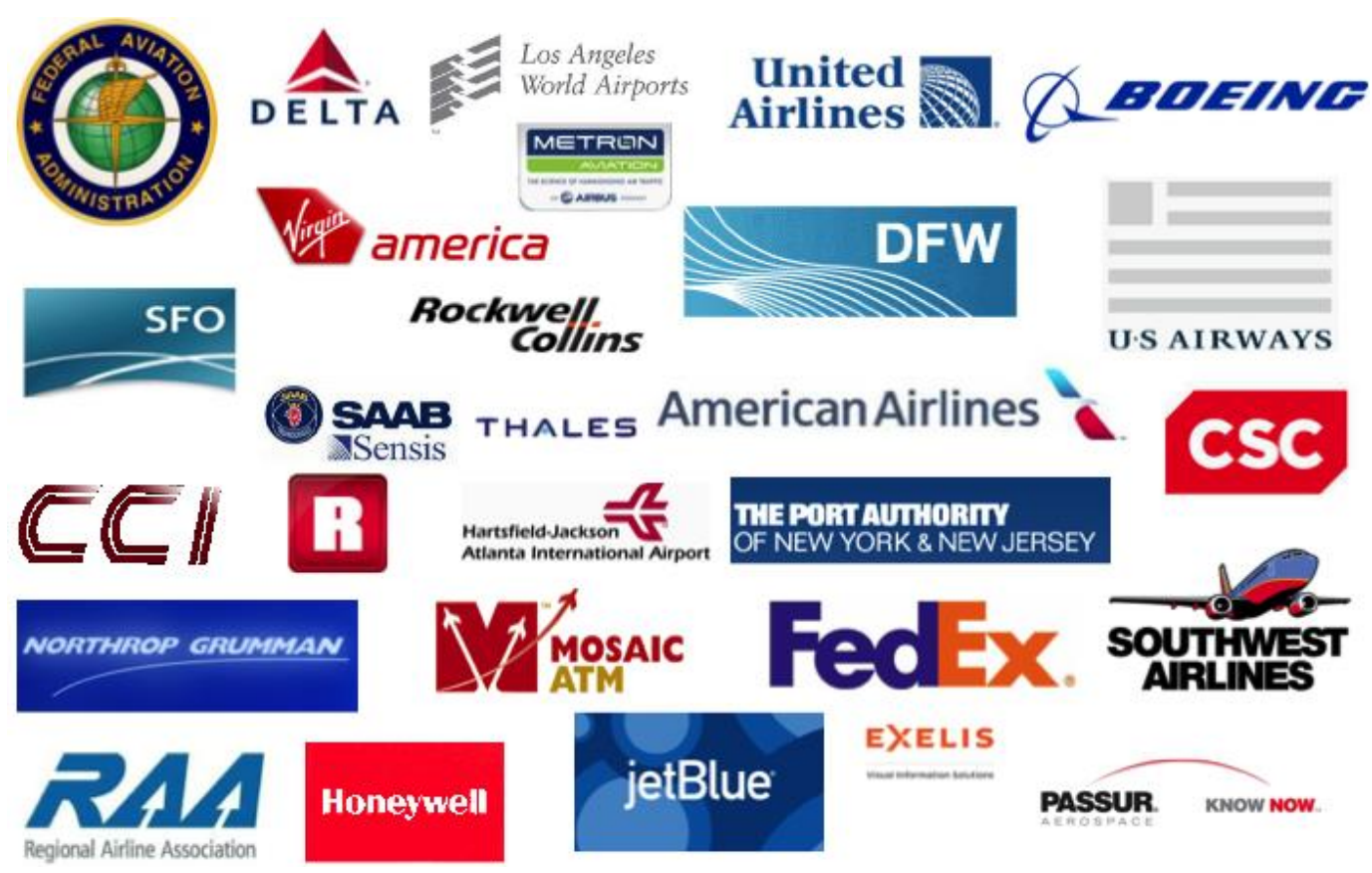

Figure 3. Stakeholders surveyed.

The survey was conducted in two phases. The first-round of the survey was designed to uncover broad issues in arrival, departure, surface operations and their impact on the stakeholders. The second-round of the survey assessed stakeholder opinion on a proposed high-level concept by NASA for IADS operations that addressed the major shortfalls identified by stakeholders in the first round. In both phases, stakeholders were interviewed in-person or by telephone. A statement on the purpose of the survey and several questions on arrival, departure, surface operations were provided prior to the interview to indicate the scope of the interview. All interviews were conducted in the February to September 2014 time frame.

To ensure consistency in the survey, a standardized set of questions was used as a basis for discussion with all stakeholders. Stakeholders were always encouraged, however, to expand the conversation as necessary to explain issues relevant to them. The standard set of questions were:

- What are your operational objectives/priorities? (i.e., what do you try to optimize and what priority do you assign to these)

- What are your key operational metrics?

- What obstacles keep you from achieving these metrics?

- What are your top-three problems in arrival/departure/surface?

- What are consequences of these problems? 
- What are your thoughts on solutions? What solutions would be most valuable and why?

- How do you determine block times and how have they changed in the past five years? (Airlines)

- What are the top three challenges to providing efficient ATC services? (ANSPs)

- What are the top three challenges to airport operations? (Airport Authorities)

\section{First-Round Stakeholder Survey Results}

In general, the stakeholders interviewed overwhelmingly supported NASA's initiative to improve IADS operations and appreciated being engaged in the process to develop a project. Several mentioned that NASA was uniquely positioned to "balance" the frequently competing interests of the stakeholders and formulate a solution that could benefit all NAS stakeholders. As a result, they provided a wealth of information on issues, experiences, and opinions. These responses were sorted, interpreted, and combined with other sources of information to arrive at the following broad issues from the point-of-view of each stakeholder category.

\section{A. Airlines}

The inability to accurately and consistently predict flight times is an important issue for airlines. This unpredictability in flight schedules adds to costs and hampers an airline's ability to plan and execute business strategy. Schedule predictability is critical to maintaining the integrity of an airline's route network with its interconnecting and interdependent flights. This predictability is also needed to efficiently utilize their primary assets, the crews and aircraft, while conforming to crew duty time and aircraft maintenance requirements and regulations.

Block time is a key metric in airline schedule and cost planning (Ref. 5). As mentioned previously, airlines have comprehensive databases of block times for routes on their network that are used to determine scheduled block times when developing future flight schedules. Although airlines probably have different methods for estimating block times, it is reasonable to assume that they all rely on historical actual block time data, incorporating "offnominal" effects such as seasonal weather effects and holiday congestion, as a basis for estimating future block times (Ref. 5). These scheduled block times are used to set flight schedules and estimate costs.

In addition to scheduled block times, an airline's published schedule specifying departure and arrival times for flights throughout its network is also based on other marketing and economic factors. Two of the factors that were identified in the survey were:

- Passenger ticket sales (revenues) are sensitive to the order in which a flight appears on a reservation screen or website when compared with the competition, as well as the preferred travel times on a particular day for a particular city pair; and

- Customer opinion (and, therefore, ticket revenue) and the airline's brand image are sensitive to an airline's on-time arrival/departure performance that is reported to the DOT and is publicly available.

As late departures and arrivals inconvenience passengers, results in negative publicity, and disrupts the integrity of the route network, airlines are conservative when estimating block times and building their schedules the more unpredictable the actual historical block time for a given city pair, the more conservative the scheduled block time that is used to set the scheduled departure and arrival times. This conservatism improves the likelihood that the flight will meet the on-time arrival performance metrics despite unanticipated delays. This conservatism is manifested as block time buffers - the more unpredictable the actual block time from experience, the greater the buffer that is applied to the scheduled block time when developing the schedule (Ref. 8). As a result, airlines have performed very well when assessed against the on-time statistics but block times have grown steadily for a majority of US city pairs over the last several decades (Refs. 14 and 15). For example, Richard Anderson, the CEO of Delta Airlines, remarked that "Delta's block time in 1956 flying between Atlanta and Washington National in a pistonengine [Douglas] DC-6 was - and is the same as it is today on a Boeing 757-200 jet" (Ref. 16).

As mentioned previously, airline operating costs scale directly with block time. The improvement in ontime performance using increased block times, therefore, results in increased cost to the airline. An FAA sponsored study estimated that block time buffers cost airlines \$3.7 billion in 2007 (Ref. 14). To manage these costs, stakeholders indicated that airline management expected operations to achieve actual block times that are a fraction (typically 65 to $75 \%$ ) of the scheduled block times.

Block time buffers also indirectly increase airline cost. The focus on being conservative by padding flight schedules increases the likelihood that flights will arrive early. Ref. 14 reports that, in 2012, 20\% of U.S. domestic 
flights by major airlines arrived 15-minutes or more ahead of their scheduled arrival time and notes that early arrivals result in increased costs due to inefficient crew and fleet utilization. Customer satisfaction can also be adversely affected when flights arrive early and cannot find an open gate, partially or completely negating the benefit of arriving early.

The Ref. 8 study dissected the cause of schedule delay and variability by examining component segments of block time. The study showed that the elapsed time for the departure phase of a flight (from OUT to OFF - see Fig. 2) is the most variable in US domestic operations and is, therefore, the most difficult to estimate. As a result, it is probably the primary cause for adding block time buffers when developing flight schedules.

Survey respondents indicated that ATC's first-come, first-served operating principle for departures is a primary factor in this uncertainty. The airline has no strategic or tactical information on the estimated OFF time prior to entering the movement area controlled by ATC. Under normal operations (i.e., when not subject to an ATCmandated traffic management program), the first time the airline is made aware of the departure queue and the estimated OFF time is when the pilot calls for taxi clearance.

The need for better long-term (strategic) and short-term (tactical) information on the status of the NAS was echoed by all the survey participants across all stakeholder categories. The airline stakeholders mentioned that there is, presently, no warning of the timing and extent of FAA Traffic Management Initiatives (TMIs) and no preview of impending NAS capacity limitations (both surface and airspace) and that improved information sharing would improve their ability to better estimate actual block times and strategically manage their schedules.

The final key issue for the airline stakeholders was the limited use of Performance Based Navigation (PBN) capability in the NAS. Many of the major domestic airlines have equipped their fleets with PBN capable avionics and the FAA has begun implementing these procedures for arrivals and departures at most airports. In practice, however, PBN procedures are not followed continuously in many airports due to several factors including airspace congestion and the presence of non-PBN equipped aircraft in the airspace. There seemed to be a consensus among the airline stakeholders that the promise of "better equipped - better served" in the NAS was not being met.

\section{B. Airports}

In an IADS context, the major operational priority for airports is maintaining throughput. These stakeholders indicated that the primary cause of loss of throughput is due to inaccurate aircraft estimated arrival times (ETAs). As the arrival stream of traffic to an airport is planned based on ETAs, late arrivals relative to these ETAs used for planning result in gaps in the arrival stream (or missed arrival "slots") that results in reduced arrival throughput. Most often, these missed slots are caused by the conservatism built into FAA mandated Ground Delay Programs (GDPs) that hold aircraft prior to departure due to a downstream constraint in the airspace or arrival airport. The decision to adopt a GDP is based on an estimation of demand at a constraint point in the airspace or destination airport using ETAs. When this demand estimation has a large degree of uncertainty (for example, if it includes a significant number of aircraft that have not yet departed and, therefore, have uncertain ETAs), the GDP will account for this uncertainty by being conservative and that can result in a loss of throughput at the destination airport.

Another major cause of loss of throughput at airports is a loss of airspace capacity due to adverse weather. Airports would like to maintain a consistent throughput regardless of weather, with the exception of extreme weather situations, through improved use of available airspace capacity.

Airport stakeholders indicated that improved data quality and decision-support tools would help alleviate potential loss of throughput. They mentioned that airport operational data was available through data sources such as the FAA's Aviation System Performance Metrics (ASPM) database but that this data was mostly unprocessed and not in a form that could be used by airports to make operational decisions. They identified a need for management tools that could help them interpret and understand the data and support decision-making.

Airports were also interested in solutions that were recognized by the FAA and widely applied across U.S. airports - they were reluctant to expend resources on solutions that were customized to a single airport or airspace that, in their experience, would not be supported by industry over the long-term.

\section{Air Navigation Service Provider}

Several groups within the FAA were interviewed including management and those involved in ATC operations. Following are the arrival, departure, surface operations issues that were identified together with ideas on implementing an eventual IADS solution:

- Reduce inter-ATC communication delays ("transactional friction") caused by manual transmission of information and approval requests by introducing intelligent data sharing (for example, coordinating 
OFF times at the departure airport to meet an available arrival slot at a destination airport via data exchange rather than voice communication).

- Use collaborative decision making capability to facilitate smooth traffic flows from smaller airports into a busy large airport in the metroplex or congested en-route airway. This will allow the ATM system to plan and schedule flows to an airport or airway over a longer time horizon, as opposed to reacting to a flight after it departs.

- Integrate existing and planned FAA capabilities to improve arrival, departure, surface operations - this refers primarily to integrating current and planned capabilities in TBFM, TFMS, and TFDM.

- Leverage on-going SWIM and TBFM/TFMS/TFDM developments to enhance the potential for implementing an IADS solution.

- Consider IADS operational improvements to alleviate congestion in busy metroplex areas (the New York terminal area, for example).

- Develop and demonstrate operational metrics that will incentivize improved operational efficiency by all stakeholders

\section{Industry}

Responses from the industry stakeholders largely reflected their individual areas of business focus and expertise. The key issues related to arrival, departure, surface operations were:

- Operations management and control processes are largely manual, high workload, and responds slowly to constraints.

- $\quad$ Need integrated solutions as opposed to the single-instance, customized, solutions at various airports that are used presently.

- $\quad$ Need improved information sharing between stakeholders, and more intelligent automation.

- $\quad$ Need improved metrics to measure NAS performance and provide incentives to the stakeholders to encourage more efficient operations.

- Improve airline schedule predictability.

- Improve airport throughput through better use of available runway capacity.

\section{Literature search on arrival, departure, surface issues and solutions}

A thorough literature search was conducted for research related to arrival, departure, and surface operations that might identify arrival, departure, surface issues as well as potential solutions. This search unearthed a broad set of issues in this area. The search spanned a multitude of references too numerous to include in this paper, therefore, an example reference is provided with each broad issue.

The key issues identified in the literature search, and their respective consequences, are:

- Lack of information sharing and inaccurate system (NAS) predictability (Ref. 17);

$>$ Status and planning information is not shared between users and ANSP's.

$>$ Unclear system status awareness leads to an inability to accurately predict its future state.

- Interfering terminal area trajectories (Ref. 18);

$>$ Usually in dense metroplex environments with common fixes and shared routes.

$>$ Poor predictability of arrival/departure demand exacerbates the problem.

- Lack of coordination of FAA Traffic Management Initiatives (TMIs) (Ref. 19);

$>$ Many TMIs are not coordinated or shared with users (some flights are affected by more than one TMI, for example).

$>$ Some TMIs are local to a particular ATC area and are not reported outside that local area.

- Manual surface operations (Ref. 20);

$>$ ATC's first-come, first-served operating paradigm leads to unpredictable behavior. 
- Lack of integration between arrival, surface, and departure operations (Ref. 21);

$>$ These operations are planned and managed independently.

- $\quad$ Lack of user/ANSP collaboration (Ref. 10);

$>$ User priorities and preferences not taken into account in arrival/departure queuing.

The issues exposed by the literature search correlated broadly with those identified in the stakeholder survey.

\section{Proposed Initial IADS Solution}

Analysis of issues identified by the stakeholder and literature surveys indicated that arresting the steady increase in block times and the related economic inefficiencies this causes would be an appropriate long-term goal for a potential IADS solution. Properly developed, implemented, and executed, such a solution would create an environment that would allow scheduled block times to trend towards their ideal unimpeded block times over the long-term. The survey information also indicated that improving the predictability and efficiency of departure operations by adopting time-based metering was the most likely mechanism to affect an improvement in scheduled block times. Three over-arching issues, outlined by the stakeholders, formed the basis for this conclusion. These were:

- Minimize variability and improve predictability of flight schedules.

- Enable more efficient routing using existing advanced navigation capabilities.

- Reduce lost airport throughput due to lack of status information and inadequate planning.

To validate this assumption, NASA developed an initial sketch of a concept that addressed this goal and discussed it with stakeholders in a second round of interviews. In addition to assessing whether the initial stakeholder feedback was understood and interpreted correctly, this second-round stakeholder survey also provided the opportunity to obtain information on the perceived benefits of the proposed solution and the characteristics and features that stakeholders perceived as being useful or essential.

The proposed solution was an efficient departure scheduler concept that will account for taxi through the top-of-climb phases of a flight. This scheduler would concurrently develop both tactical and strategic solutions with appropriate levels of fidelity and confidence. The strategic scheduler would develop an optimized schedule that balances airport and airspace demand and capacity over a long time horizon (roughly 20-min or more prior to pushback) while the tactical scheduler would adjust the schedule closer to departure (roughly 20-min or less prior to pushback) to account for actual variations in the schedule (delayed flights, unplanned airport constraints, etc.). The goal of the strategic scheduler would be to provide an overall awareness of airport operations by developing the expected departure sequence at the runway and other relevant points on the surface over a long time horizon. Inputs to the strategic scheduler would include flight ready times provided by airlines, arrival information, airport capacity, taxi routes and speeds, surface surveillance, traffic management initiative restrictions, overhead stream traffic and departure fix restrictions for merging and de-confliction, wake vortex separation restrictions, and airport/runway configuration. Outputs would include de-conflicted target take-off times and associated target airport movement area entry times and intermediate times at other appropriate points on the airport surface. The strategic scheduler would facilitate collaborative planning with airline operators to allow schedules to be adjusted when needed. The goal of the tactical scheduler component would be to enable uninterrupted taxi/climb trajectories while maintaining surface/terminal area throughput in all traffic conditions. This scheduler's primary goal would be to reduce flight time, fuel burn, noise, and emissions by using proven optimization methods to facilitate largely unimpeded departure operations from the gate to top-of-climb. The overall scheduling concept would build on relevant existing scheduling technologies and concepts of operation.

\section{Second-Round Stakeholder Survey Questions}

A subset of the stakeholders from the first-round survey, composed primarily of airlines and organizations that provide arrival, departure, surface operations-related services and products to the aviation industry, was approached for the second-round survey. A description of the proposed departure scheduler concept was provided to the stakeholders (with the level of detail provided in the description above) together with the following set of standard questions (as before, the stakeholders were encouraged to expound on any question or expand the discussion beyond these questions, if desired):

- Would this departure scheduler concept be useful to your organization - why or why not? 
- What do you see as the most beneficial features of such a scheduler and how would these benefit your organization?

- What features are essential to a departure scheduler?

- What is the downside to a scheduler (i.e., what must it not do)?

- What metrics would your organization use to assess the value of such a scheduler?

- NASA has a relatively short time-frame (five years) to develop and demonstrate the feasibility of this concept - what are the minimum features should be included in a demonstration that would give you confidence that the new concept will be useful?

- How would you like to participate in developing and demonstrating this concept?

- Additional Questions for Vendors of existing schedulers:

What are the inputs to your scheduler?

$>$ What do you optimize on in your scheduler?

$>$ What do you output from your scheduler?

$>$ What would you like to add to improve the optimization of your scheduler?

\section{Results of the Second-Round Survey}

The key results of the second-round stakeholder survey were:

- All the stakeholders surveyed unanimously agreed that a departure scheduler would benefit the NAS, for the following reasons:

$>$ It would improve schedule predictability that is of paramount importance to airlines.

$>$ It would reduce taxi-times and fuel use.

$>$ NASA's "holistic" (integrated) approach was better than the many custom solutions currently available.

$>$ Moving to a time-based paradigm on the surface is critical to improving IADS (change from the current first-come, first-served paradigm).

$>$ It promises to improve schedule predictability.

- The stakeholders indicated that the scheduler should exhibit the following characteristics:

$>$ Timeliness - provide necessary information reasonably in advance (to facilitate strategic and tactical decision-making).

$>$ Logical - users can have confidence in the information the system provides and will accept its recommendations.

$>$ Accuracy - information provided is repeatable and reliable;

$>$ Flexibility - should incorporate user/stakeholder preferences and input;

> Robustness - it should adapt reliably (with repeatability) to unexpected events; and

$>$ Transparency/equitability - its operating principles and performance are available to all stakeholders and are clearly understood.

- The scheduler must work in metroplex environments with multiple airports in relatively close proximity. It should:

$>$ Incorporate airspace constraints and allow alternate/efficient departure routes.

$>$ Be transportable; i.e., it must work at different airports with different airport systems.

- The stakeholders maintained that both strategic and tactical components of the scheduler were equally important - the strategic would facilitate long-term planning with less precision and the tactical would give them a preview of near-term adjustments to the schedule.

- It should provide decision support to ATC on departure scheduling while allowing flexibility when necessary.

- This concept would be a valuable enhancement to the FAA's terminal and surface management programs. 
- The concept's potential to synergistically integrate the FAA's surface and terminal area traffic management system upgrade programs was of particular interest.

\section{Conclusions}

NASA surveyed NAS stakeholders to understand the key issues and potential solutions in IADS operations. The intent was to use this information to formulate a project that would develop and demonstrate a solution that maximized benefit to all the stakeholders. Four stakeholder groups were surveyed - they were: airlines, airports, airnavigation service providers, and industry service/product providers. An initial survey indicated that improving the predictability and efficiency of departure operations by adopting time-based metering would improve the predictability of flight schedules, and decrease costs and fuel use. In a follow-on survey, all the stakeholders supported a departure scheduling concept that could enable unimpeded transit from gate-to-cruise in a busy metroplex airspace environment, and would allow stakeholders to share information and express schedule preferences through a collaborative decision-making process. The stakeholders agreed that this proposed solution would improve flight schedule predictability, facilitate better strategic planning for airlines and airports, and benefit

all stakeholders. The stakeholders also outlined the characteristics that the departure scheduler should exhibit in order to benefit IADS operations. The results of this stakeholder survey are being used to formulate the goals for NASA's ATD-2 sub-project that is presently in an advanced planning stage.

\section{Acknowledgements}

The authors greatly appreciate the contributions of the following: Robert Rosen, Matthew Blake, and others at Crown Consulting, Inc., for their support of the stakeholder and literature surveys; Capt. Robert Koteskey of San Jose State University for explaining the intricacies of airline crew operations; and most importantly, the stakeholders who enthusiastically responded to the survey and devoted their time to educate the NASA team on IADS operations and economics.

\section{References}

1. Federal Aviation Administration, "NextGen Implementation Plan,” June 2013.

2. Swenson, H. N., Hoang, T., Engelland, S., et al, "Design and Operational Evaluation of the Traffic Management Advisor at the Fort Worth Air Route Traffic Control Center," Presented at the 1st USA/Europe Air Traffic Management Research \& Development Seminar, Saclay, France, June, 1997.

3. Coppenbarger, R., Hayashi, M., et al, "The Efficient Descent Advisor: Technology Validation and Transition," AIAA 2012-5611, Presented at the 12th AIAA Aviation Technology, Integration, and Operations (ATIO) Conference and 14th AIAA/ISSM, September 2012, Indianapolis, Indiana.

4. Swenson, H., Robinson, J. E. III, and Winter, S., "NASA's ATM Technology Demonstration 1 - Moving NextGen Arrival Concepts from the Laboratory to the Operational NAS," Journal of Air Traffic Control, Summer 2013, Volume 55, No. 2, pp. 27-37.

5. Centeno, M., and Vitt, U., "Chip Off The Old Block,” Ascend Magazine, 2011 Issue No. 2.

6. Midkiff, A. H., Hansman, J. R., and Reynolds, T. G., "Air Carrier Flight Operations," MIT International Center for Air Transportation, Report No. 2004-3, July 2004.

7. "Airline Metric Concepts for Evaluating Air Traffic Service Performance," Report of the CNS/ATM Focused Team, Air Traffic Services Performance Focus Group, Feb. 1999.

8. Skaltsas, G., Belobaba, P., and Cohn, A., "Flight Time Components and Their Delays on Domestic Routes," Presented at the MIT Global Airline Industry Program - IAB/Industry Consortium Joint Meeting, Nov. 2010.

9. Engelland, S.A., Capps, A., Day, K., Kistler, M., Gaither, F., and Juro, G., "Precision Departure Release Capability (PDRC) Final Report,” NASA/TM-2013-216533, June 2013.

10. Federal Aviation Administration, "TFDM Core for ATCTs - Concept of Operations," FAA Terminal Flight Data Manager (TFDM) Program Office, ConOps PMO-02-TFDM-13-001, Rev. 2.1, Nov. 2013.

11. Federal Aviation Administration, "System Wide Information Management (SWIM)," www.faa.gov/nextgen, Dec 2014. 
12. Simons, M., "A Functional Analysis of Integrated Arrival, Departure, Surface Operations in NextGen," Presented at the 31st Digital Avionics Systems Conference, Williamsburg, VA, October, 2012.

13. Howell, D., and McInerney, T., "Estimating the Achievable Benefits of Airport Surface Metering," AIAA 2012-5653, Presented at the 12th AIAA Aviation Technology, Integration, and Operations (ATIO) Conference and 14th AIAA/ISSM Conference, September 2012, Indianapolis, Indiana.

14. Frank, T., "Airlines pad flight schedules to boost on-time records," USA TODAY, Feb. 15, 2013.

15. McCartney, S., "Why a Six-Hour Flight Now Takes Seven," Wall Street Journal, Feb. 4, 2010.

16. Anderson, R., "Future of the Aviation Industry," Remarks to the U.S. Chamber of Commerce, Washington, D.C., April 12, 2012.

17. Malik, W., Gupta, G., and Jung, Y.C., "Managing Departure Aircraft Release for Efficient Airport Surface Operations," Presented at the AIAA Modeling and Simulation Technologies Conference, Toronto, Canada, Aug. 2010.

18. DeLaura, R. A., Underhill, N. K., Hall, L. M., and Rodriguez, R. G., "Evaluation of the Integrated Departure Route Planning (IDRP) Tool 2011 Prototype, MIT Lincoln Laboratories, Project Report ATC-388, May 2012.

19. Airports Council International, "Airport Collaborative Decision Making - Enhancing Airport Efficiency," 2012.

20. Srivastava, A., "Improving Departure Taxi Time Predictions Using ASDE-X Surveillance Data," Proceedings of the $30^{\text {th }}$ IEEE/AIAA Digital Avionics Systems Conference, 2011.

21. Diffenderfer, P., Tao, Z., and Payton, G., "Automated Integration of Arrival/Departure Schedules," Tenth USA/Europe Air Traffic Management Research and Development Seminar (ATM2013), June 2013. 